\title{
Increased aperiodic gamma power in young boys with Fragile $X$ Syndrome is associated with better language ability
}

\author{
Carol L. Wilkinson * (i) and Charles A. Nelson
}

\begin{abstract}
Background: The lack of robust and reliable clinical biomarkers in Fragile X Syndrome (FXS), the most common inherited form of intellectual disability, has limited the successful translation of bench-to-bedside therapeutics. While numerous drugs have shown promise in reversing synaptic and behavioral phenotypes in mouse models of FXS, none have demonstrated clinical efficacy in humans. Electroencephalographic (EEG) measures have been identified as candidate biomarkers as EEG recordings of both adults with FXS and mouse models of FXS consistently exhibit alterations in resting state and task-related activity. However, the developmental timing of these EEG differences is not known as thus far EEG studies have not focused on young children with FXS. Further, understanding how EEG differences are associated with core symptoms of FXS is crucial to successful use of EEG as a biomarker, and may improve our understanding of the disorder.

Methods: Resting-state EEG was collected from FXS boys with full mutation of Fmr1 (2.5-7 years old, $n=11$ ) and compared with both age-matched $(n=12)$ and cognitive-matched $(n=12)$ typically developing boys. Power spectra (including aperiodic and periodic components) were compared using non-parametric cluster-based permutation testing. Associations between 30 and $50 \mathrm{~Hz}$ gamma power and cognitive, language, and behavioral measures were evaluated using Pearson correlation and linear regression with age as a covariate.

Results: FXS participants showed increased power in the beta/gamma range $(\sim 25-50 \mathrm{~Hz})$ across multiple brain regions. Both a reduction in the aperiodic (1/f) slope and increase in beta/gamma periodic activity contributed to the significant increase in high-frequency power. Increased gamma power, driven by the aperiodic component, was associated with better language ability in the FXS group. No association was observed between gamma power and parent report measures of behavioral challenges, sensory hypersensitivities, or adaptive behaviors.

Limitations: The study sample size was small, although comparable to other human studies in rare-genetic disorders. Findings are also limited to males in the age range studied.

Conclusions: Resting-state EEG measures from this study in young boys with FXS identified similar increases in gamma power previously reported in adults and mouse models. The observed positive association between resting state aperiodic gamma power and language development supports hypotheses that alterations in some EEG measures may reflect ongoing compensatory mechanisms.
\end{abstract}

Keywords: Fragile X Syndrome, Electroencephalography, Biomarker, Gamma, E:I ratio, Outcome measures, Language

${ }^{*}$ Correspondence: Carol.wilkinson@childrens.harvard.edu Division of Developmental Medicine, Boston Children's Hospital, 1

Autumn Street, 6th Floor, Boston, MA 02115, USA

\section{Introduction}

Fragile X Syndrome (FXS), an X-linked, single-gene disorder, is the most common inherited form of intellectual disability [1]. In addition to cognitive deficits, 
children with FXS often have significant language impairments as well as behavioral challenges that overlap with several neurodevelopmental disorders including autism spectrum disorder (ASD), attention deficit hyperactivity disorder (ADHD), and anxiety[2]. Indeed, virtually all boys with FXS present with some autistic symptoms, and 30-50\% meet formal diagnostic criteria for ASD [3, 4]. Therefore, understanding the neural mechanisms that underlie specific cognitive, language, and behavioral deficits in FXS is both crucial to the development of effective therapeutics, but also may shed light on the pathophysiology of comorbid disorders.

The disorder is caused by an expansion of a CGG trinucleotide repeat on the $\mathrm{X}$ chromosome, leading to silencing of the Fmr1 gene, and reduced expression of its protein product, Fragile X Mental Retardation Protein (FMRP) [2]. Although research in rodent models of FXS has improved our understanding of the molecular mechanisms underlying the disorder, there is still no effective treatment for FXS. In addition to mimicking many of the cognitive and behavioral phenotypes seen in humans, the Fmr1 KO mice have alterations in synaptic and structural plasticity, including impairments in long-term potentiation and depression [5], and alteration in excitatory/ inhibitory (E:I) balance [6-9]. Whereas several compounds (eg. mGluR5 negative modulators [10], GABA agonists [11, 12]) have successfully reversed phenotypes in Fmr1 KO mice, human phase II trials have disappointingly shown limited effect on outcome measures [13]. A lack of brain-based biomarkers in FXS, and other ASDrelated disorders has been identified as a major challenge to therapeutic development in the field [13-15].

Electroencephalography (EEG) is a brain-based biomarker candidate, as it is low cost, non-invasive, and has been used in both human and mouse studies of FXS. EEG studies in both FXS adults and Fmr1 KO mice have observed alterations in resting state power as well as reduced habituation to repeated auditory stimuli (termed repetition suppression) [16-23]. Indeed reduced repetition suppression has been associated with worse nonverbal cognitive scores in FXS adults, and medication trials in FXS have used EEG measures of repetition suppression to monitor treatment effects [21,24]. Specific alterations in gamma oscillation have also been consistently observed; FXS adults and Fmr1 KO mice have increased resting-state gamma band power compared to controls, and reduced inter-trial phase synchrony in the gamma band in response to auditory stimuli [17, 19, 20, 25]. Human studies with FXS individuals have also observed reduced relative alpha power and elevated relative theta power [16-18] compared to neurotypical individuals, but this has not been consistently observed in mouse studies.
Aberrant gamma oscillations are particularly intriguing as a brain-based biomarker for FXS and overlapping neuropsychiatric disorders for several reasons. First, gamma oscillations are generated by parvalbumin-expressing inhibitory interneurons, and thereby indirectly represent E:I balance in the cortex [26]. Alterations in both inhibitory neurons and gamma oscillations have been observed not only in FXS [27], but several other neuropsychiatric/ neurodevelopmental disorders including schizophrenia and ASD [28-33]. Second, gamma activity has been associated with clinically relevant processes such as sensory integration, language processing [34-38] and working memory $[39,40]$. Further, alterations observed in the gamma band in Fmr1 KO mice have been rescued by targeted pharmacological intervention [25].

However, it is still unclear how observed alterations in the gamma band relate to core cognitive and behavioral features of FXS, especially in the developing brain. In a recent, relatively large $(n=38)$ EEG study in adults with FXS [16], increased frontal gamma power $(30-80 \mathrm{~Hz})$ was significantly associated with a number of behavioral features reported on the Aberrant Behavioral Checklist (eg. irritability, hyperactivity, stereotyped behaviors), a commonly used parent report measure in clinical trials. In addition gamma power was negatively associated with adaptive measures of communication and a direct measure of cognition. Similarly, increased gamma power prior to speech production has also been observed in FXS individuals, and is associated with reduced intelligibility and low verbal IQ [41]. Smaller studies in FXS adults have also found associations between resting-state gamma power and increased sensory sensitivity and social impairment [17]. Similarly, in our study of toddlers with familial risk of ASD, we have found increased frontal gamma power $(30-50 \mathrm{~Hz})$ to be negatively associated with language development [36], and in boys with ASD increased gamma power has been associated with worse developmental delay [42].

To our knowledge no published studies have characterized baseline EEG activity in young boys with FXS. This is an important gap in the field, as therapeutics (both behavioral and pharmacologic) will ideally be implemented near the age of diagnosis ( 3 years of age). In addition, only a few studies have specifically looked at how differences in brain activity relate to core features of FXS-cognitive and language impairments [16, 21, 41]. Given these gaps, this study had two main goals. First, we aimed to characterize baseline EEG activity in a group of preschool to young school aged boys with full-mutation FXS, as compared to either age-matched or cognitivematched typically developing boys. We hypothesized that FXS boys would have similar baseline EEG abnormalities observed in FXS adults with increased theta and gamma 
power. Second, we aimed to characterize the relationship between baseline EEG measures and a range of cognitive measures. This was done in both a hypothesis driven and exploratory manner. We tested two hypotheses based on previous findings: (1) gamma power $(30-50 \mathrm{~Hz})$ would be positively associated with various behavioral challenges as measured by the Aberrant Behavior Checklist and (2) gamma power would be negatively associated with cognition and language ability. Given that no prior studies have evaluated EEG and related symptoms in this age range, exploratory analyses investigated possible associations between gamma power and additional parent report measures often used in clinical trials.

\section{Methods}

\section{Participants}

A total of 16 boys (33-78 months old) with full mutation of Fmr1 and 12 similarly aged (32-80 months old) typically developing boys were recruited for this study (IRB\#P00025493) conducted at Boston Children's Hospital/Harvard Medical School. 4 FXS participants did not complete baseline EEG acquisition (3 net refusal, 1 unable to maintain protocol), and 1 FXS participant's EEG data was excluded due to excessive artifact. EEG and behavioral data were analyzed for a total of 11 FXS boys (mean age $=53.5$ months; $\mathrm{SD}=16.4$ months, range 33-78 months old) and 12 typically developing boys (mean age $=47.7$ months; $\mathrm{SD}=13.1$, range $32-80$ months old).

FXS and age-controls: FXS participants all had documented full mutation of the Fmrl gene, but could have size mosaicism (mixture of full and premutation) and methylation status was not known for all participants. Girls were excluded from this study given their variable expression of Fmrl and the small size of this study. Across all groups, additional exclusion criteria included history of prematurity ( $<35$ weeks gestational age), low birth weight $(<2000 \mathrm{~g})$, known birth trauma, known genetic disorders (other than FXS), unstable seizure disorder, current use of anticonvulsant medication, and uncorrected hearing or vision problems. Some participants were on stable doses of medications (Oxybutin (1 age-matched control); melatonin (2 FXS); Miralax (1 agematched control). Children were from primarily Englishspeaking households with English spoken more than 50\% of the time (2/11 FXS and 2/12 age-matched control participants were either in bilingual households or daycare).

Cognitive and sex-matched controls: EEG data from an additional set of 12 cognitive-matched boys were analyzed (mean age $=29.8$ months; $\mathrm{SD}=10.1$ months, range 14-52 months old). 11 individuals from this group provided EEG data as part of a concurrent longitudinal study (IRB\#P00018377) in the lab which used the same EEG resting-state paradigm. Exclusion criteria were the same as above. In addition infants in this group did not have a sibling with autism. Controls were identified by matching FXS participants' Fine Motor and Visual Reception raw scores on the Mullen Scales of Early Learning (see below). In order to appropriately match all FXS participants, one EEG in this group overlapped with the above age/sex matched control group. Given this, age vs cognitive matched controls were not statistically compared.

Institutional review board approval was obtained prior to starting the study. Written, informed consent was obtained from all parents or guardians prior to their children's participant in the study. Table 1 describes participant characteristics.

\section{EEG assessment}

Resting-state EEG data were collected in a dimly lit, sound-attenuated, electrically shielded room. The child either sat in their seated caregiver's lap or sat independently in a chair, high-chair, or stroller based on behavioral preference. Caregivers were instructed by a research assistant to avoid social interactions or speaking with their child. Continuous EEG was recorded for 2-5 min depending on compliance. Given known behavioral challenges in the FXS population, parents were asked about expected behavioral challenges, calming techniques, and motivators specific to each child. To improve compliance, participants were shown a silent screensaver of abstract colorful moving images and allowed to hold a fidget toy. EEG data were collected using a 128-channel Hydrocel Geodesic Sensor Net (Version 1, EGI Inc, Eugene, OR) connected to a DC-coupled amplifier (Net Amps 300, EGI Inc, Eugene, OR). Data were sampled at $1000 \mathrm{~Hz}$ and referenced to a single vertex electrode $(\mathrm{Cz})$, with impedances kept below $100 \mathrm{k} \Omega$ in accordance with the impedance capabilities of the high-impedance amplifiers inside the electrically shielded room [43]. Electrooculographic electrodes were removed to improve the child's comfort.

\section{EEG pre-processing}

Raw NetStation (NetStation version 4.5, EGI Inc, Eugene, OR) files were exported to MATLAB (version R2017a) for pre-processing and power analysis using the Batch EEG Automated Processing Platform (BEAPP; [44]) with integrated Harvard Automated Preprocessing Pipeline for EEG (HAPPE; [45]). Preprocessing has previously been described in detail for similar data [36]. Briefly, data were $1 \mathrm{~Hz}$ high-pass and $100 \mathrm{~Hz}$ low-pass filtered, downsampled to $250 \mathrm{hz}$, and then run through the HAPPE module for $60 \mathrm{~Hz}$ line noise removal, bad channel rejection and artifact removal using combined wavelet-enhanced independent component analysis (ICA) and Multiple Artifact Rejection 
Table 1 Sample characteristics

\begin{tabular}{|c|c|c|c|}
\hline & $\begin{array}{l}\text { Age-matched } \\
N=12\end{array}$ & $\begin{array}{l}\text { FXS } \\
\mathrm{N}=11\end{array}$ & $\begin{array}{l}\text { Cog-matched } \\
\mathrm{N}=12\end{array}$ \\
\hline Age, mean in months (SD) & $47.6(13.1)$ & $53.5(16.3)$ & $29.8(10.1)$ \\
\hline \multicolumn{4}{|l|}{ Maternal education, $n(\%)$} \\
\hline$<4$-year college degree & $0(0)$ & $0(0)$ & $1(8)$ \\
\hline 4-year college degree & $4(33)$ & $2(18)$ & $2(17)$ \\
\hline > 4-year college degree & $8(77)$ & $9(81)$ & $9(75)$ \\
\hline \multicolumn{4}{|l|}{ Paternal Education, n (\%) } \\
\hline$<4$-year college degree & $0(0)$ & $1(9)$ & $3(25)$ \\
\hline 4-year college degree & $6(50)$ & $4(36)$ & $6(50)$ \\
\hline > 4-year college degree & $6(50)$ & $6(54)$ & $3(25)$ \\
\hline \multicolumn{4}{|l|}{ Household income, $n(\%)$} \\
\hline$<\$ 40,000$ & $0(0)$ & $0(0)$ & $1(8)$ \\
\hline$\$ 40-70,000$ & $0(0)$ & $3(27)$ & $0(0)$ \\
\hline$\$ 70-100,000$ & $3(25)$ & $3(27)$ & $0(0)$ \\
\hline$\$ 100-140,000$ & $5(42)$ & $1(8)$ & $4(33)$ \\
\hline$>\$ 140,000$ & $4(33)$ & $4(36)$ & $7(58)$ \\
\hline \multicolumn{4}{|l|}{ Race, $n(\%)$} \\
\hline White & $7(58)$ & $9(81)$ & $9(75)$ \\
\hline African American & $0(0)$ & $0(0)$ & $1(8)$ \\
\hline Asian & $1(8)$ & $1(9)$ & $0(0)$ \\
\hline Mixed & $4(33)$ & $1(9)$ & $2(17)$ \\
\hline \multicolumn{4}{|l|}{ Ethnicity, $n(\%)$} \\
\hline Hispanic or Latino & $0(0)$ & $3(27)$ & $1(8)$ \\
\hline \multicolumn{4}{|l|}{ Mullen Scales of Early Learning } \\
\hline Nonverbal Developmental Quotient (Mean \pm SD) & $112.6 \pm 15.7$ & $58.2 \pm 14.3$ & $102.8 \pm 16.2$ \\
\hline Visual Reception Age Equiv (months \pm SD) & $54.5 \pm 8.8$ & $32.3 \pm 14.4$ & $32.2 \pm 13.3$ \\
\hline $\begin{array}{l}\text { Fine Motor Age Equiv } \\
\text { (months } \pm \text { SD) }\end{array}$ & $44.6 \pm 7.1$ & $29.5 \pm 12.0$ & $29.5 \pm 12.0$ \\
\hline \multicolumn{4}{|l|}{ EEG Quality Measures } \\
\hline Number of $2 \mathrm{~s}$ Segments & $116.9 \pm 17.4$ & $82.1 \pm 38.5$ & $101.9 \pm 34.4$ \\
\hline Percent Good Channels & $94.9 \pm 4.0$ & $94.1 \pm 3.6$ & $90.6 \pm 3.8$ \\
\hline Percent ICs Rejected & $36.2 \pm 7.6$ & $34.0 \pm 9.1$ & $37.0 \pm 10.0$ \\
\hline Median Artifact Probability & $0.03 \pm 0.02$ & $0.07 \pm 0.04$ & $0.05 \pm 0.05$ \\
\hline
\end{tabular}

Algorithm (MARA [46, 47]). Given the short length of EEG recording, 39 of the 128 channels were selected for ICA/MARA (Standard 10-20 electrodes: 22, 9, 33, 24, $11,124,122,45,36,104,108,58,52,62,92,96,70,83$; Additional electrodes: 23, 28, 19, 4, 3, 117, 13, 112, 37, $55,87,41,47,46,103,98,102,75,67,77,72$ ). These electrodes were chosen based on their spatial location, covering frontal, temporal, central, and posterior regions of interest for later analysis (see Fig. 1). After artifact removal, channels removed during bad channel rejection were interpolated, data were rereferenced to an average reference, detrended using the signal mean, and segmented into 2-s segments. Any segments with retained artifact were rejected using HAPPE's amplitude and joint probability criteria.
EEG were rejected for data quality if they had fewer than 20 segments ( $40 \mathrm{~s}$ total), or did not meet the following HAPPE data quality output parameters: percent good channels $>80 \%$, mean and median retained artifact probability $<0.3$, percent of independent components rejected $<84 \%$, and percent variance after artifact removal $<32 \%$. Table 1 shows quality metrics for all groups.

\section{EEG power analysis}

Power spectral density (PSD) at each electrode, for each $2 \mathrm{~s}$ segment, was calculated with multitaper spectral analysis [48] embedded in BEAPP using three orthogonal tapers. For each electrode for a given EEG, PSD for each frequency bin $(0.5 \mathrm{~Hz}$ frequency resolution) was 

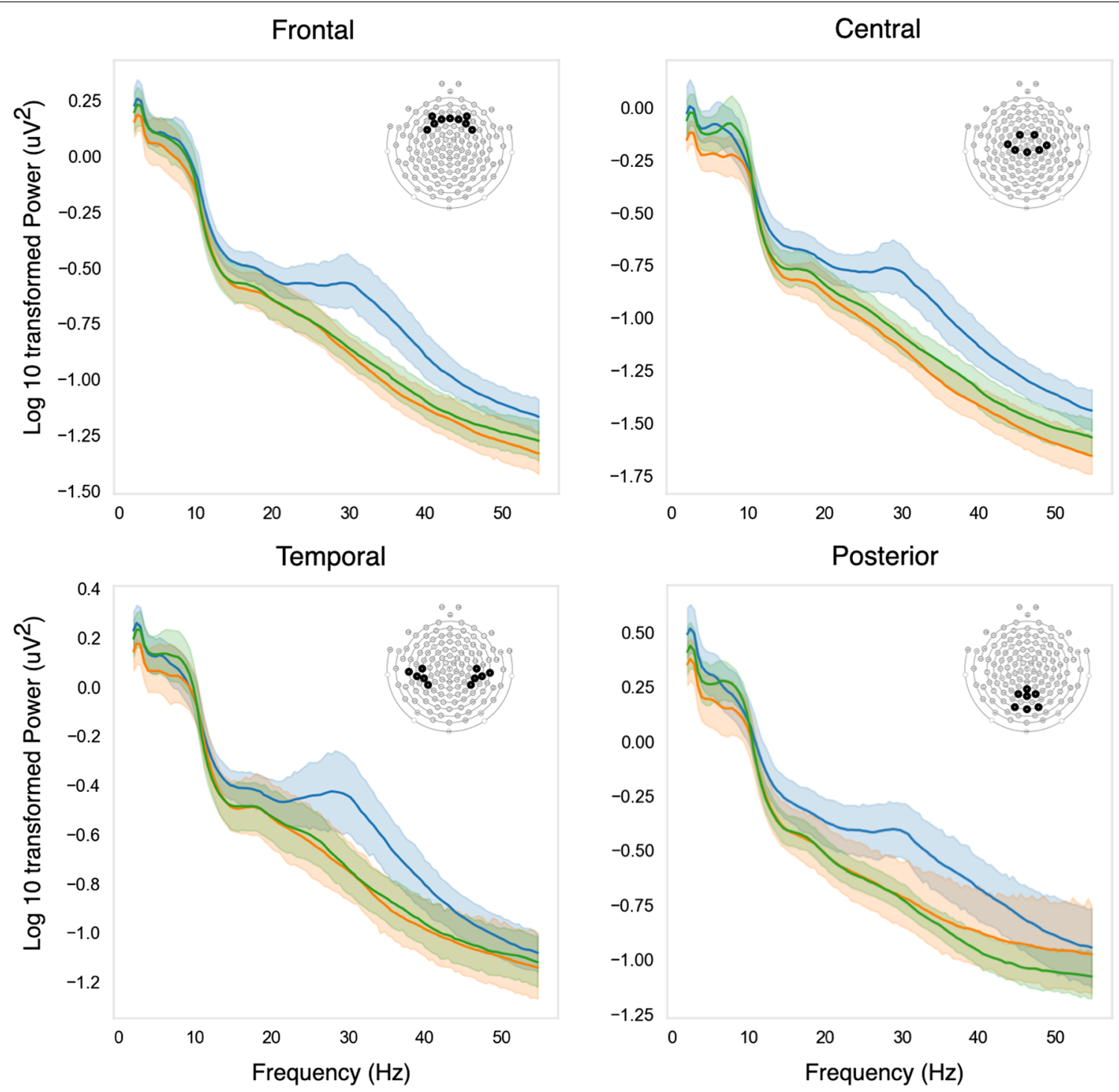

FXS Age-Matched

Cognitive-Matched

Fig. 1 Absolute power spectra across frontal, central, temporal, and posterior regions of interest. Log 10 transformed power spectra of FXS (blue), age-matched controls (green), and cognitive-matched controls (orange) are shown for each region of interest. Shaded areas describe $95 \%$ confidence intervals

averaged across segments, and then averaged across the regions of interest shown in Fig. 1. PSDs were normalized using $\log 10(\mathrm{~Hz})$. Our analyses were limited to absolute power, as relative power measurements were artificially affected by normalization; increased power in high frequency bands in FXS participants artificially lowered the relative power of lower frequency bands. The PSD was also analyzed using the FOOOF v1.0.0 parameterization model across a $2-55 \mathrm{~Hz}$ frequency range (https://githu b.com/fooof-tools/fooof; in Python v3.6.8) in order to model periodic and aperiodic components of the power spectra [49]. The FOOOF model was used in the fixed mode (no spectral knee) with peak_width_limits set to $[1,18.0]$, max_n_peaks $=7$, and peak_threshold =2). For each subject's power spectrum FOOOF provides two parameters to describe the aperiodic 1/f background signal: offset and slope. To determine an aperiodic-adjusted gamma power, the FOOOF estimated aperiodic signal was subtracted from the raw power spectrum, resulting in a flattened spectrum. FOOOF model fit to the original 
spectrum for each group is shown in Additional file 1: Figure 1.

\section{Behavioral Measures}

The Mullen Scales of Early Learning (MSEL [50]) is a standardized cognitive measure for children 0-69 months of age. Non-verbal subscales (fine motor, visual reception) were administered to all FXS participants regardless of age, age-matched controls under 70 months of age, and all cognitive matched controls, and the Nonverbal Developmental Quotient (NVDQ) was calculated. The Preschool Language Scale 5th Edition (PLS [51]), a comprehensive developmental language assessment standardized for children 0-83 months of age, was administered to FXS and age-matched participants. The PLS was used instead of the MSEL to assess language, as it covers the full age range of the study sample and has been recently updated to include toys and images that are more consistent with items children interact with today. Standard scores of subtests for receptive (Auditory Comprehension) and expressive (Expressive Communication) language, as well as the total standard score were calculated. Note that per research administration protocol augmentative communication devices are not used during this assessment, so scores may underestimate a child's non-verbal expressive language skills. The following clinical questionnaires were completed by primary caregivers of FXS and age-matched participants: Aberrant Behavior Checklist-Community Edition [52] (ABC-FXS, scored using FXS specific factoring system [53]), Vineland Adaptive Behavior Scales, Third Edition(VABS-3 [54], Repetitive Behavior Scale-Revised [55], and Sensory Profile, Child-2 [56]. The ABC-FXS scoring included 6 subscales: irritability, hyperactivity, lethargy, social avoidance, stereotypy, and inappropriate speech.

\section{Statistical Analyses}

T-test, or Mann Whitney if data was not normal in distribution, was used to compare differences in behavioral scores or EEG measures between either FXS versus agematched controls, or FXS versus cognitive-matched controls. To examine group differences in the power spectra, a non-parametric clustering method, controlling for multiple comparisons using Monte Carlo estimation (1000 permutations) [57] was employed with MNE-Python [58] using a F-statistic threshold of 4.32. Regression analysis was used to characterize the relationship between gamma power and behavioral measures within the FXS group. Analysis were performed used Stata software, version 14.2 (Stata). Figures were created using Python v3.6.8 and python data visualization libraries [matplotlib [59] and Seaborn (https://seaborn.pydata.org/index.html)].

\section{Results}

Sample description

Demographic data, including the MSEL non-verbal developmental quotient (NVDQ) and subtest age-equivalents are shown in Table 1. As expected, FXS participants had significantly lower nonverbal skills compared to age-matched controls (t-tests; MSEL NDVQ: $p<0.0001$, Fine Motor: $p<0.01$, Visual Reception: $p<0.001$ ), but had similar age-equivalent scores to the cognitive-matched control group. Groups were also similar on EEG quality metrics and well below our quality thresholds (see methods). However the average number of EEG segments available for power analyses was significantly lower in the FXS group compared to age-matched (Mann Whitney, $p<0.05$ ), but not cognitive-matched control groups (Mann Whitney, $p>0.05$ ).

\section{Power spectra}

Power spectra for all three participant groups across frontal, temporal, central, and posterior regions of interest are shown in Fig. 1. FXS power spectra differences were most prominent in the beta-gamma range compared to both age-matched controls and cognitive-matched controls across all regions of interest. A non-parametric clustering method, controlling for multiple comparisons, was used to identify significant differences in the power spectra (Table 2). Analysis of the frontal power spectra, identified a cluster between 23 and $55 \mathrm{~Hz}$ for FXS versus age-matched controls $(p=0.004)$, and between 22 and $50 \mathrm{~Hz}$ for FXS vs cognitive-match controls $(p=0.007)$ with significant group differences. Similar clusters were identified for the central power spectra. In contrast, clusters identified for temporal power spectra were smaller in range $(26-40 \mathrm{~Hz})$, with $p$ values at, or just below the critical alpha (0.05). For posterior power spectra, the cluster identified between FXS and age-match controls was not significant, however between FXS and cognitively-match controls a cluster between 19 and $47 \mathrm{~Hz}$ was identified $(p=0.014)$. Several lower frequency clusters were also identified however none of these were significantly different between groups.

Visually, the power spectra of FXS participants also appear to have a reduced slope, suggesting that both aperiodic and oscillatory activity are altered. Growing evidence suggests that aperiodic activity (defined as $1 / \mathrm{f}^{\mathrm{x}}$, with $x=$ slope of the aperiodic curve) in part represents broad neural firing and balance of excitation and inhibition [60]. Further aperiodic activity changes with age and with behavior $[61,62]$, underscoring its likely functional role in cognition. To better understand differences in the aperiodic and oscillatory power spectra components in FXS participants, we used the Fitting Oscillations and 
Table 2 Power spectra clusters with significant group differences

\begin{tabular}{|c|c|c|c|c|}
\hline \multirow[t]{2}{*}{ Region of interest } & \multicolumn{2}{|c|}{ FXS versus age-matched } & \multicolumn{2}{|c|}{ FXS versus cognitive-matched } \\
\hline & Frequency range $(\mathrm{Hz})$ & Cluster $p$ value & Frequency range $(\mathrm{Hz})$ & Cluster $p$ value \\
\hline Frontal & $22.9-54.7$ & 0.004 & $22.0-50.3$ & 0.007 \\
\hline Frontal & $12.7-15.6$ & 0.188 & - & - \\
\hline Central & $19.0-54.7$ & 0.003 & $21.5-52.2$ & 0.009 \\
\hline Central & $2.9-5.9$ & 0.196 & - & - \\
\hline Central & $12.2-18.6$ & 0.132 & - & - \\
\hline Temporal & $25.9-40.0$ & 0.041 & $26.4-39.6$ & 0.053 \\
\hline Posterior & $23-55$ & 0.073 & $11.7-15.6$ & 0.147 \\
\hline Posterior & - & - & $22-50$ & 0.014 \\
\hline
\end{tabular}

Clusters with significant differences between groups are bolded

One-Over-F (FOOOF) algorithm [49] to estimate aperiodic $1 / \mathrm{f}$ (Fig. 2a) and periodic/oscillatory components (Fig. 2b) of the power spectra. Individual power spectra tracings are showing in Additional file 1: Figure 2. There were no significant difference in the offset of the aperiodic power spectrum in the FXS group ( $p^{\prime} \mathrm{s}>0.4$; Fig. $2 \mathrm{c}$, $d$, Additional file 1: Figure 3). The observed trend in reduced aperiodic slope for the FXS group compared to age-matched controls was marginally significant for frontal $(p=0.13)$ and central $(p=0.07)$ regions. Permutation cluster analysis of the frontal aperiodic-adjusted power spectra identified a cluster between $28-41 \mathrm{~Hz}$ for FXS versus age-matched controls $(p=0.003)$, and between 26 and $43 \mathrm{~Hz}$ for FXS versus cognitive-match controls $(p=0.005)$ with significant group differences. Similar significant clusters were identified for the other regions of interest. Further analysis of this frequency range found both a significant shift in frequency peak frequency and significant increase in peak amplitude for the FXS group compared to age-matched and cognitive matched controls (FXS: $\mathrm{Hz}=31.1 \pm 3.72, \mu \mathrm{V}=0.31 \pm 0.16$; agematched: $\mathrm{Hz}=25.6 \pm 4.28, \mu \mathrm{V}=0.18 \pm 0.11$ cognitivematched: $\mathrm{Hz}=24.2 \pm 4.09, \mu \mathrm{V}=0.16 \pm 0.06$; Fig. $2 \mathrm{e}$, f). An additional cluster in the theta/alpha range $(\sim 5-10 \mathrm{~Hz})$ was also identified for central, temporal, and posterior regions of interest, however $p$ values were marginally significant $(p=0.08-0.13$; Additional file 1 : Table 1.)

\section{Frontal gamma power and associations with clinical outcomes}

Given previous studies in adults with FXS demonstrating significant increase in frontal gamma power and associations with various clinical outcome measures, we focus our following analyses on this frequency band alone. A priori to the cluster analyses described above, we hypothesized that frontal gamma power $(30-50 \mathrm{~Hz})$ would be increased in FXS children, and that frontal gamma power would be positively associated with behavioral challenges as measured by the Aberrant Behavior Checklist and negatively associated with cognition and language ability. Power in the gamma frequency band was calculated in two ways. First, PSD calculations between 30 and $50 \mathrm{~Hz}$ were averaged to determine average gamma power for each group (Fig. 3a). FXS participants had significantly higher gamma power $(-0.87 \pm 0.16 \mu \mathrm{V})$ compared to age-matched $(-1.12 \pm 0.16 \mu \mathrm{V} ; p=0.001)$ and cognitivematched $(-1.08 \pm 0.14 \mu \mathrm{V} ; p=0.003)$ controls. Given group differences in the number of EEG segments analyzed, we confirmed that gamma power was not associated with number of EEG segments retained (Adjusted $R^{2}=0.006$; B Coefficient $=-0.001, p=0.3$ ). Second, we calculated aperiodic and periodic components of gamma power, using the FOOOF estimated aperiodic and aperiodic-adjusted power spectra shown in Fig. 2a, b. Both the aperiodic and aperiodic-adjusted gamma were significantly elevated in FXS participants compared to age- and cognitive-matched comparison groups (Fig. 3b, c).

We next investigated whether increased frontal gamma power in FXS participants was associated with three clinical measures: (1) parent report on the Aberrant Behavior Checklist (ABC-FXS), (2) non-verbal cognitive skills measured by the Mullen Scales of Early Learning (MSEL) nonverbal developmental quotient, and (3) language development as measured by the Preschool Language Scale-5 (PLS-5) as well as parent report on the Vineland Adaptive Behavior Scale (VABS-3, Communication subscale).

There was no significant correlation between frontal gamma power and scores on any of the six ABC-FXS subscales (Pearson $R$ range: -0.24 to 0.19 ), or with the MSEL nonverbal developmental quotient (Pearson $R$ : 0.16). In contrast, there was a significant and unexpected positive correlation with large effect size between frontal gamma power and standard scores on language measures (Fig. 4) both based on behavioral 

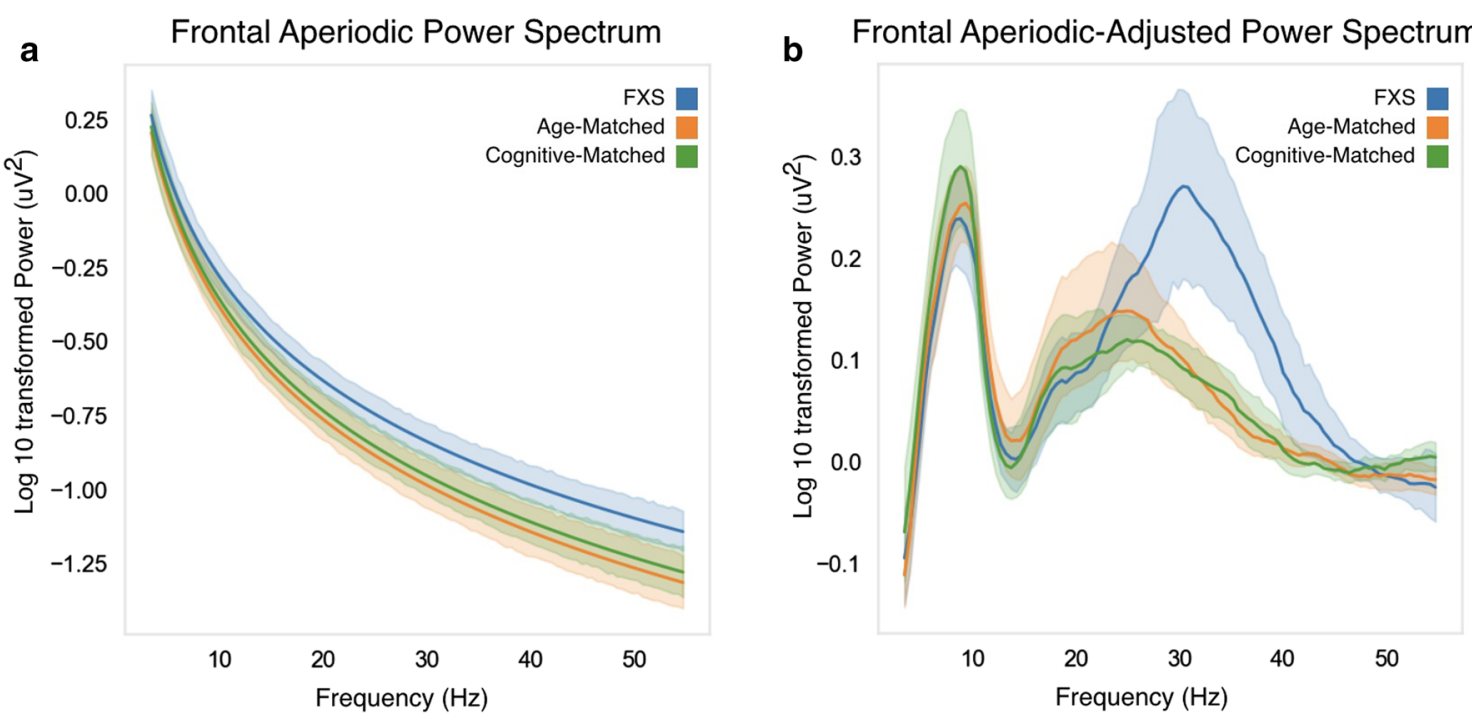

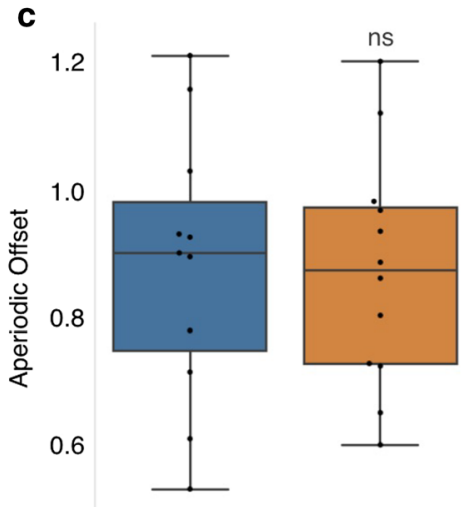

0.4
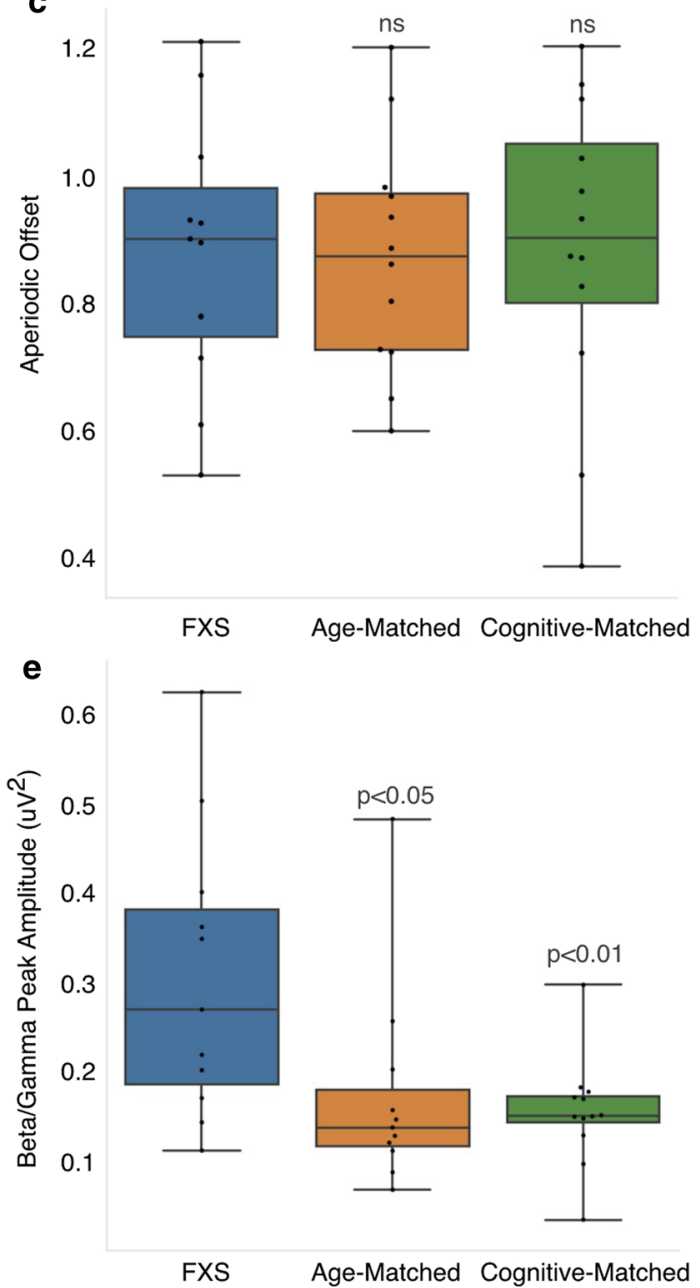

d
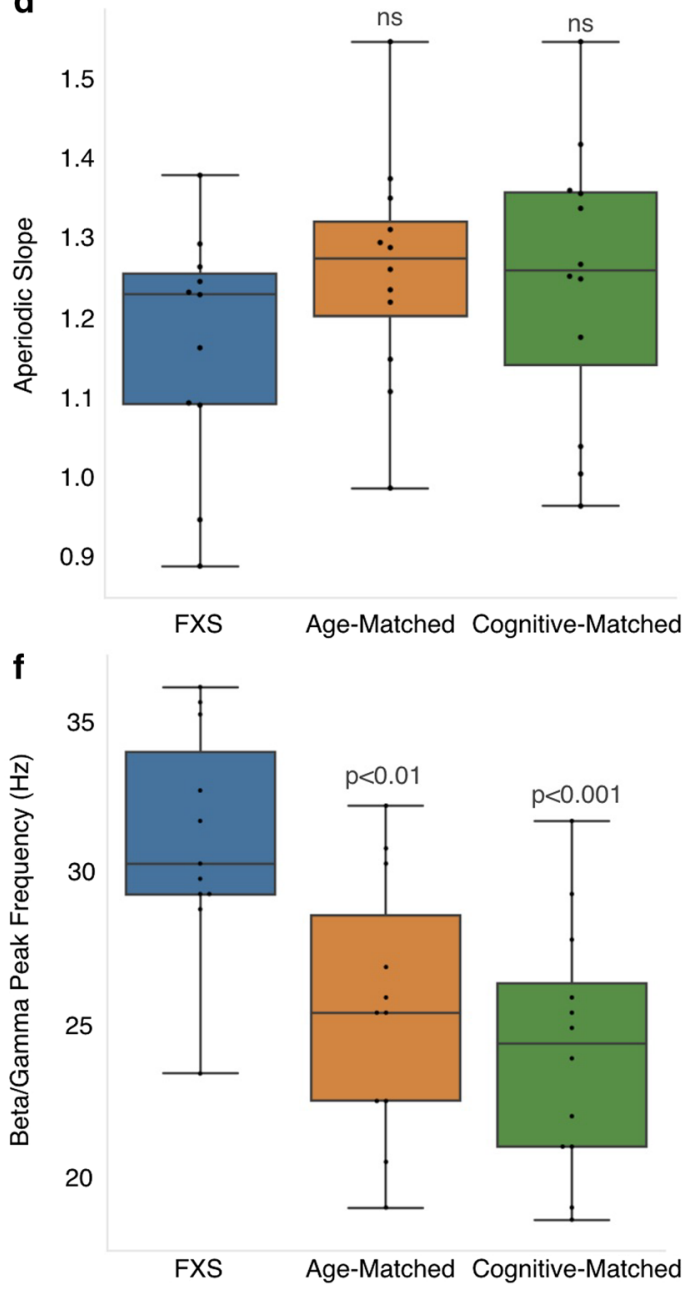

Fig. 2 a FOOOF estimated aperiodic power spectrum FXS (blue), age-matched controls (green), and cognitive-matched controls (orange). b FOOOF estimated aperiodic power spectrum was subtracted from the raw power spectrum to determine aperiodic-adjusted power spectrum. Shaded areas describe $95 \%$ confidence intervals. c FOOOF estimated aperiodic offset. d FOOOF estimated aperiodic exponent. e Amplitude of maximal peak from Aperiodic-Adjusted Power Spectra between 15 and $40 \mathrm{~Hz}$. f Frequency of identified maximal peak. $p$ values represent t-test comparisons with FXS group 

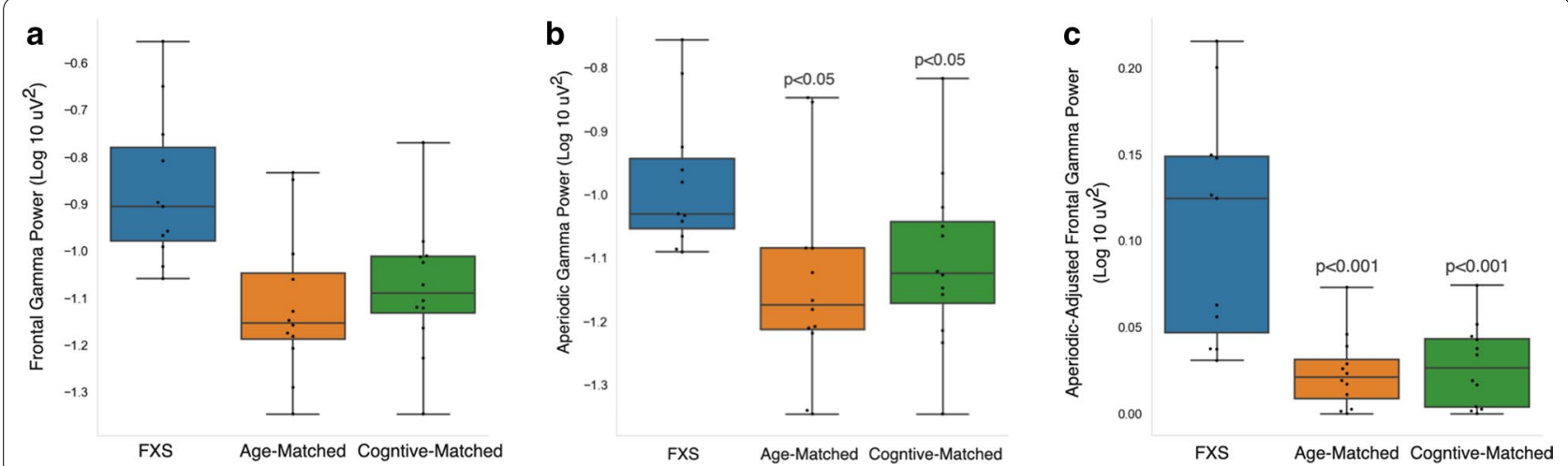

Fig. 3 Power in the gamma frequency band $(30-50 \mathrm{~Hz}$ ) derived from either the $\mathbf{a}$ frontal power spectra shown in Fig. 1a, b aperiodic frontal power spectra shown in Fig. 2a, or c aperiodic-adjusted frontal power spectra shown in Fig. 2b. $p$ values represent t-test comparisons with the FXS group
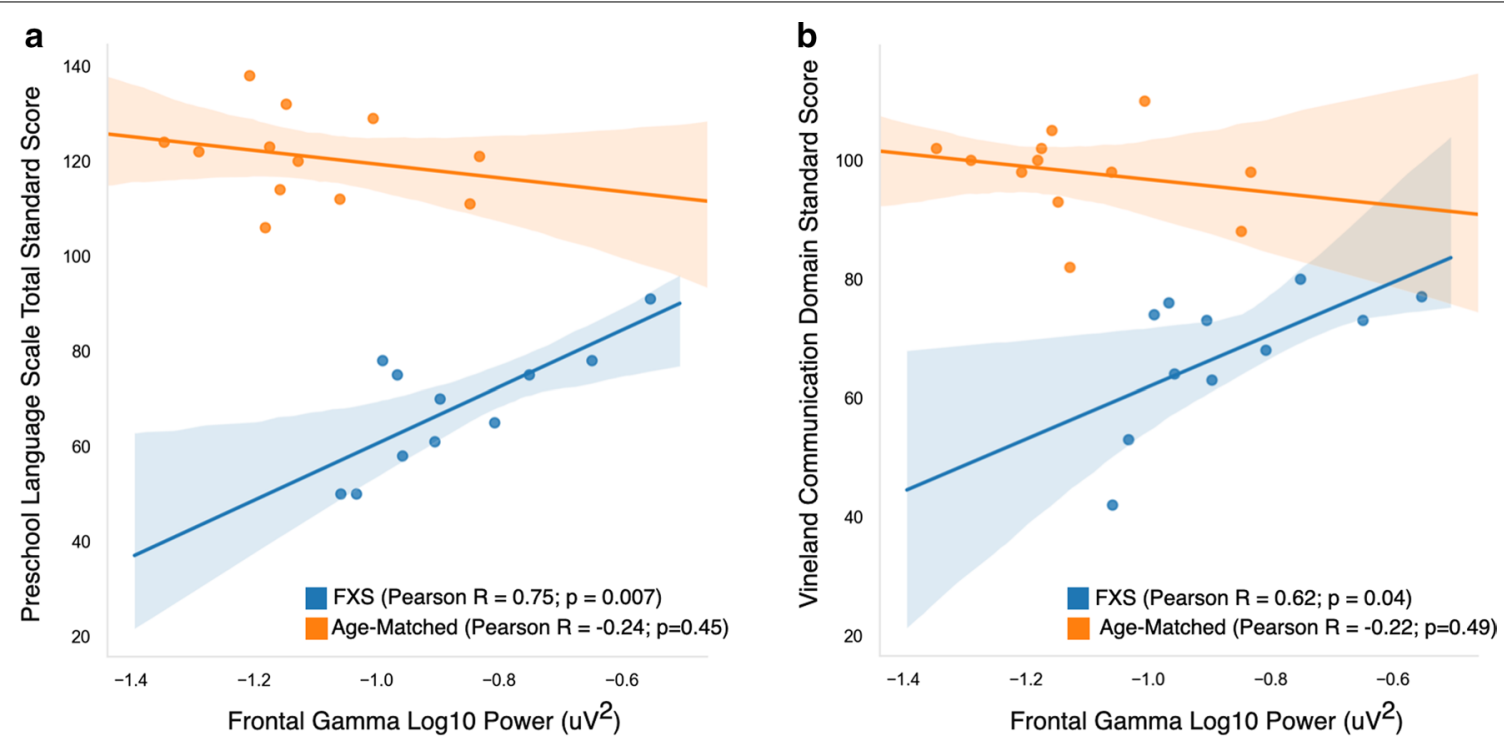

Fig. 4 Correlation graphs between overall frontal gamma power $(30-50 \mathrm{~Hz})$ and $\mathbf{a}$ total standard score on the Preschool Language Scale; and b standard score on the communication domain of the Vineland Adaptive Behavior Scale

assessment (PLS-5; Pearson $R=0.75 ; p=0.007$ ) and parent report (VABS-3, Communication Subscale; Pearson $R=0.62 p=0.04)$. Increased gamma power was associated with better language skills. There was no observed relationship between frontal gamma power and language in the age-matched comparison group. Given the large age range of participants, and the possibility that age may influence both standard scores and gamma power, a linear regression analyses were performed with age (in months) included as a covariate (Table 3). The positive association between gamma power and language ability as measured by the PLS-5, but not the VABS-3, remained significant after adjusting for age.
To further dissect the functional relevance of increased aperiodic and periodic gamma activity in FXS participants, we also investigated the relationship between aperiodic and periodic/oscillatory components (labeled aperiodic-adjusted) of the gamma band and these two language measures. Notably, the association between gamma and PLS-5 Total Standard Score appears to be driven by the aperiodic component of the gamma band (Fig. 5). The adjusted $R^{2}$ was low in models using the aperiodic-adjusted gamma (Table 4), whereas models using the aperiodic component of gamma had high adjusted $R^{2}$ values, and the positive association between aperiodic gamma and the PLS-5 scores remained significant when age was included in the model. While there 
Table 3 Linear regression models of frontal gamma power and language measures

\begin{tabular}{|c|c|c|c|c|}
\hline \multirow[t]{2}{*}{ Dependent variable } & \multicolumn{2}{|c|}{ Preschool Language Scale } & \multicolumn{2}{|c|}{ VABS Communication Subscale } \\
\hline & Model 1 & Model 2 & Model 1 & Model 2 \\
\hline Adjusted $R^{2}$ & 0.52 & 0.47 & 0.32 & 0.34 \\
\hline \multicolumn{5}{|c|}{ Variables [B coefficient (SE)] } \\
\hline Intercept & $120.2(15.2)$ & $120.3(16.1)$ & $105.8(16.3)$ & $105.1(16.0)$ \\
\hline Frontal gamma power & $59.6(17.3)^{* *}$ & $64.5(23.6)^{*}$ & $43.9(18.5)^{*}$ & $26.1(23.4)$ \\
\hline Age (months) & - & $0.08(.23)$ & - & $-0.28(.23)$ \\
\hline
\end{tabular}

${ }^{*} p<0.05$

${ }^{* *} p<0.01$
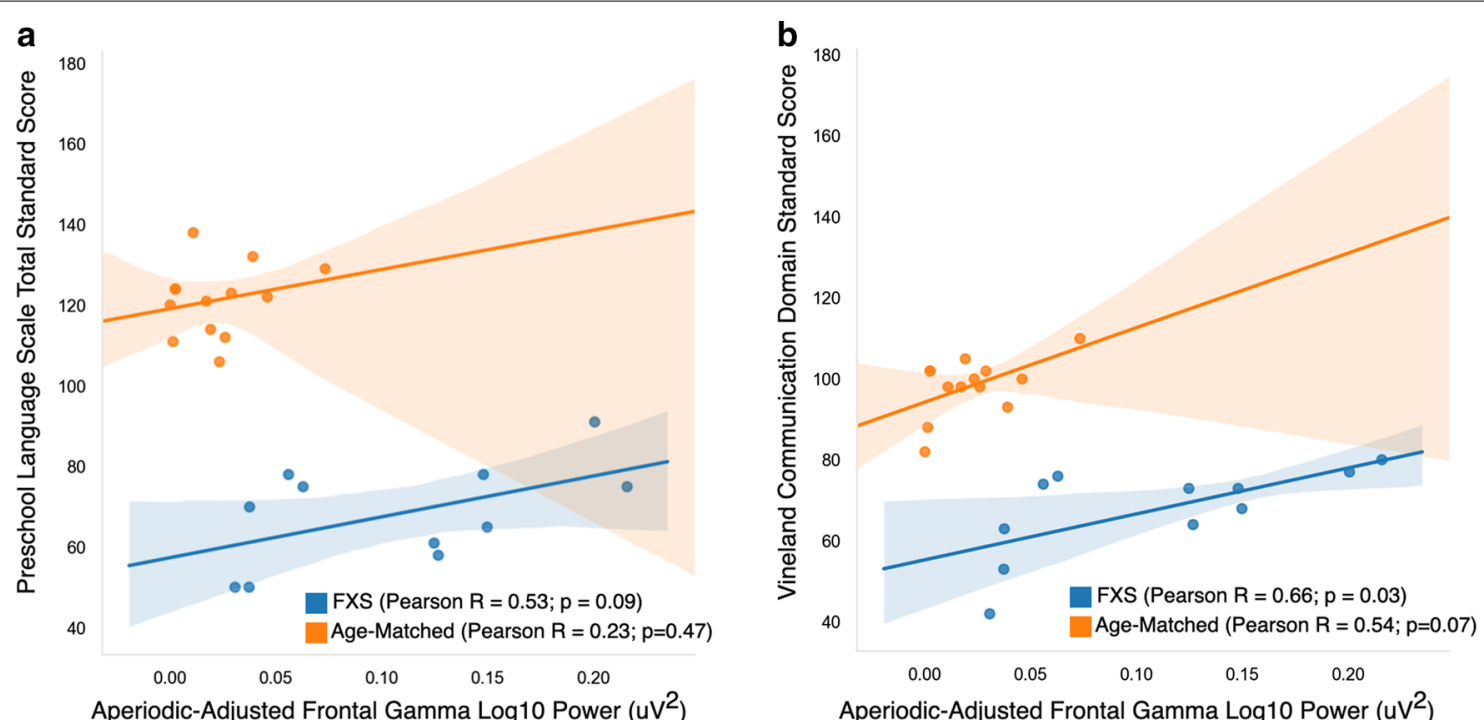

Aperiodic-Adjusted Frontal Gamma Log10 Power $\left(\mathrm{uV}^{2}\right)$

Aperiodic-Adjusted Frontal Gamma Log10 Power $\left(\mathrm{uV}^{2}\right)$
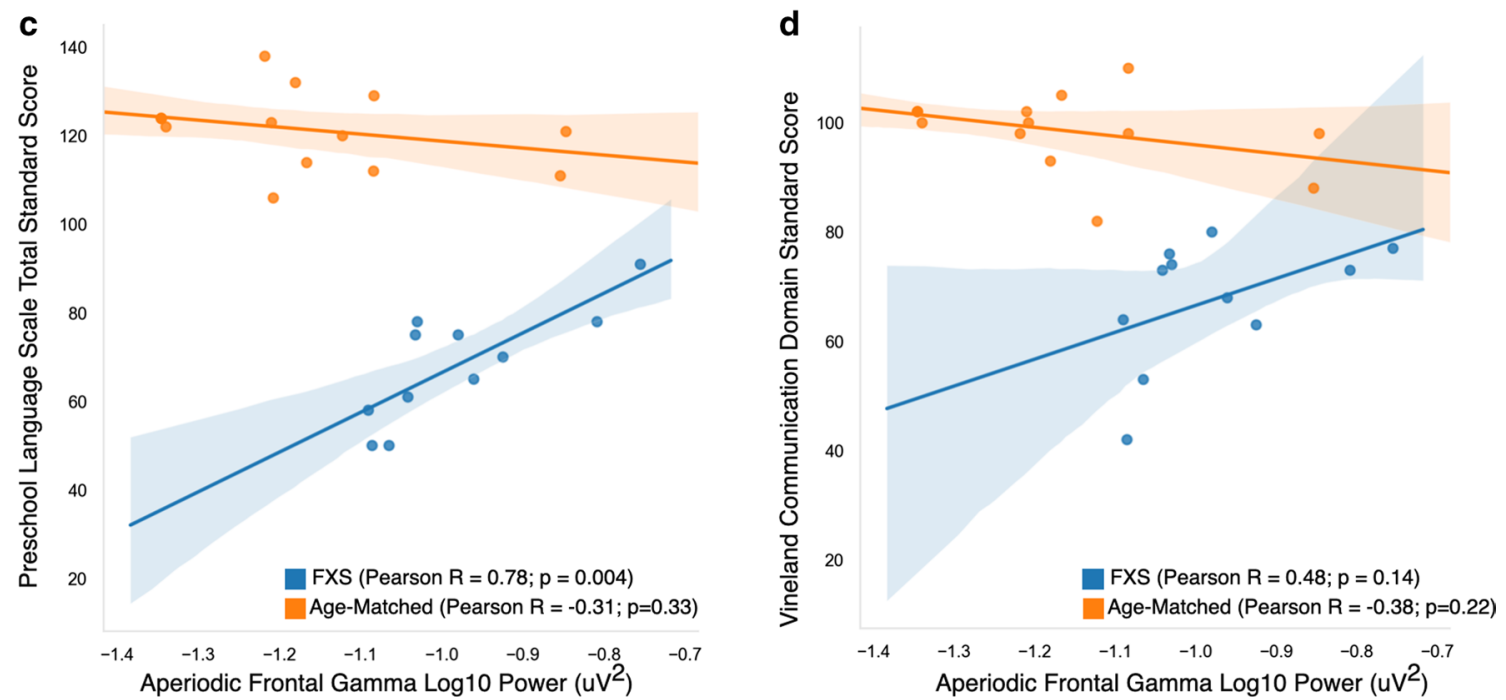

Fig. 5 Top (a, b): Correlation graphs between aperiodic-adjusted frontal gamma power and language measures. Bottom (c, d): Correlation graphs between aperiodic frontal gamma power and language measures 
Table 4 Linear Regression FOOOF estimated frontal gamma power measures and language

\begin{tabular}{|c|c|c|c|c|}
\hline & \multicolumn{2}{|c|}{ Preschool Language Scale } & \multicolumn{2}{|c|}{ VABS Communication Subscale } \\
\hline & Model 1 & Model 2 & Model 1 & Model 2 \\
\hline Adjusted $R^{2}$ & 0.20 & 0.1 & 0.37 & 0.33 \\
\hline \multicolumn{5}{|l|}{ Variables [B coefficient (SE)] } \\
\hline Intercept & $57.3(6.8)$ & $56.4(30.3)$ & $55.3(5.4)$ & $69.8(23.4)$ \\
\hline Aperiodic-adjusted gamma & $101.4(54.0)$ & $103.7(96.3)$ & $113.5(42.8)^{*}$ & $75.4(74.4)$ \\
\hline \multirow[t]{2}{*}{ Age (months) } & - & $0.1(0.39)$ & - & $-0.19(0.3)$ \\
\hline & Model 1 & Model 2 & Model 1 & Model 2 \\
\hline Adjusted $R^{2}$ & 0.56 & 0.53 & 0.14 & 0.33 \\
\hline \multicolumn{5}{|l|}{ Variables [B coefficient (SE)] } \\
\hline Intercept & $156.3(23.8)$ & $156.3(24.6)$ & $115.8(29.9)$ & $115.8(26.3)$ \\
\hline Aperiodic gamma & $89.8(24.2)^{* *}$ & $83.2(26.8)^{*}$ & $49.2(30.3)$ & $29.1(28.8)$ \\
\hline Age (months) & - & $-0.12(0.18)$ & - & $-0.37(0.20)$ \\
\hline
\end{tabular}

${ }^{*} p<0.05$

${ }^{* *} p<0.01$

was a significant positive correlation between aperiodicadjusted gamma and the VABS-3 Communication Subscale standard score, this did not survive adjustment for age. Post-hoc analyses revealed that age was highly correlated with aperiodic-adjusted gamma (Pearson $R=-0.80 ; p=0.003$ ) but was not correlated with either aperiodic gamma band activity (Pearson $R=-0.37$; $p=0.27$ ) or with aperiodic slope (Pearson $R=0.16$ ) (Additional file 1: Figure 4).

Additional exploratory analyses of clinical correlations found no significant associations with parent report measures of sensory hypersensitivity as measured by the Sensory Profile-2, other adaptive subscales on the VABS-3 (e.g. daily living, social skills, motor skills), or repetitive behaviors as reported on the Repetitive Behavior Scale (Graphs and Pearson Correlations Coefficients shown in Additional file 1: Figure 5).

\section{Discussion}

In this study we compare resting (non-task related) EEG power spectra of young boys with FXS to both age-matched and cognitive-matched typically developing boys. Consistent with previous studies in both adolescents and adults with FXS, as well as mouse models of FXS, we observed an increase in power from roughly $25-50 \mathrm{~Hz}$ across multiple brain regions. Previous studies in adults have also reported increases in resting-state theta power. In this study, permutation cluster analysis identified a single cluster crossing the theta band in the central electrodes, however this cluster did not meet statistical significance. Further, we observed that the increase in high-frequency power is the result of both a decrease in the slope of the aperiodic signal and an increase in high-frequency $(\sim 25-40 \mathrm{~Hz})$ activity. Surprisingly, within the FXS group, increased high-frequency activity (as measured by the gamma band $30-50 \mathrm{~Hz}$ ) was associated with better language ability.

While the sample size of this study is small, it is the first EEG study focused on preschool to young school aged boys with FXS. Acquiring high quality EEG data from this younger population is challenging, however it is crucial to understanding how underlying neuropathophysiology in FXS changes across development, and to identifying biomarkers that reflect specific core impairments. Notably, identified differences between FXS power spectra and both age-matched and cognitive matched comparison groups were very similar, suggesting that these differences are specific to the genetic disorder, and not due to differences in age or cognitive ability.

\section{Aperiodic power spectra findings}

The measured power spectrum from EEG is the result of a mixture of signals including an aperiodic, broadband background signal, and narrowband oscillatory components. Here we observe that FXS participants have a reduced aperiodic slope, but similar aperiodic offset, leading to overall significantly increased aperiodic activity in the higher frequency bands. Computational modeling suggests that changes in the aperiodic slope are in part driven by the balance between excitation (E) and inhibition (I), with an increased E:I ratio leading to a reduced slope [60]. This is consistent with hyperexcitable states observed in Fmr1 KO mice [6, 8, 63], and evidence of inhibitory dysfunction in FXS [6, 7, 27, 64]. Further, elegant experiments by Antoine et. al. [9] observed increased E:I ratios in the primary somatosensory cortex 
of four ASD-mouse models, including Fmr1 KO mice. Interestingly, increased E:I ratios were not accompanied by expected increased firing rates or increased postsynaptic potentials in layer $2 / 3$ neurons. Indeed, in vivo recordings from awake Fmr1 KO mice showed no changes in spontaneous firing of regular spiking layer $2 / 3$ neurons and reduced whisker-evoked firing. The authors hypothesize the observed increased E:I ratio represents compensation for altered cortical spiking in order to normalize firing rates. This raises the question of whether increased E:I ratios are functionally beneficial to individuals with FXS. Our language findings support this hypothesis.

Based on previous studies in adults with FXS, we originally hypothesized that increases in gamma power in FXS participants would be negatively associated with language development. However, if increased aperiodic gamma activity reflects homeostatic compensation for ongoing altered cortical spiking resulting in an E:I imbalance, but normalized firing rates, then we may expect improved language development in individuals with the most appropriate homeostatic compensation, especially at a young age. Here we demonstrate that gamma power, specifically the aperiodic signal in the gamma range, was positively associated with language development. Further, model fit was quite strong in our linear regression models limited to aperiodic gamma with and without age as a covariate, with more than $50 \%$ of the variance of PLS-5 language scores explained. However, model fit was notably worse for predicting scores on the VABS-3 Communication Subscale, and associations between gamma power and VABS-3 scores were not significant once age was included in the model. A similar pattern was also observed for the VABS-3 Social Subscale that had moderate, but not significant, associations with frontal gamma power (Additional file 1: Figure 5) prior to adjustment for age. Statistically, given the increased variance in the relationship VABS-3 Communication and gamma power in the context of our low sample size, we may not be sufficiently powered to identify a significant relationship in this measure. Further, VABS-3 is a parent report measure that focuses more on functional communication skills that can also be impacted by additional factors such as social interest and anxiety. Therefore high-frequency aperiodic activity may specifically be relevant to language development. Indeed, aperiodic gamma power was not associated with non-verbal cognitive skills as measured on the MSEL, or parent report of attention, irritability, sensory hypersensitivity or repetitive/stereotyped behaviors.

The specificity of the association between aperiodic gamma activity and language, instead of for example overall cognition is perhaps surprising, given the growing view that aperiodic or broadband gamma activity reflects overall levels of cortical activity, rather than responses to specific sensory stimuli. However, in light of the findings from Antoine et al. in FXS mice discussed above, it is possible that the increase in aperiodic gamma is more reflective of the balance of excitatory and inhibitory inputs than overall increase in cortical firing. Such underlying imbalances may in turn affect tuning of sensory inputs [65]. We hypothesize that a reduced aperiodic slope, or increased aperiodic gamma, may be associated with improved gamma-phase locking during language or auditory tasks specifically for the preschool age. This is in contrast to what has been observed in FXS adult and Fmr1 KO EEG studies, where increased background gamma activity is associated with reduced intertrial phase synchrony in the gamma band in response to a modulated auditory "chirp" stimulus [66, 67]. However, such associations may change over development; Wen and colleagues have found enhanced evoked gamma responses in juvenile (P21) and adult (P60) Fmr1 KO mice, but reduced responses at P30. Additional studies are needed to evaluate the relationship between aperiodic gamma and evoked gamma in this younger age range.

The positive relationship between gamma power and language in young boys with FXS also contrasts with our previous study in toddlers with familial risk of autism, where we observed a negative relationship between gamma power and language development. Differences in findings may be related the younger age of the infantsibling study, as well as differences in participants characteristics; Boys with full mutation of $F m r 1$ will all have developmental delays, whereas this is a risk but not a certainty for infants with ASD siblings. However, there were similarities in the pattern of compensation, in that both FXS boys and high familial risk toddlers, better language was associated with more atypical gamma power for the given participant group (high familial risk toddlers have lower gamma power compared to controls). Future investigation of even younger FXS participants, as well as FXS children with mosaicism will be valuable to further understanding these complexities.

\section{Aperiodic-adjusted power spectra findings}

We also observed increased power between 25 and $40 \mathrm{~Hz}$ in the periodic component of the power spectra for FXS participants, with an oscillatory peak observed in most individual tracings (Additional file 1: Figure 2) at the border of the canonical beta/gamma frequency bands $(\sim 30 \mathrm{~Hz})$. This peak was both significantly higher in amplitude and shifted from 25 to $30 \mathrm{~Hz}$ compared to both age- and cognitive-matched participants. Aperiodic-adjusted gamma overall was not strongly associated with language measures. This may reflect several 
variables. First, in our study sample aperiodic-adjusted gamma was strongly negatively correlated with age, making associations with other variables more difficult given our sample size. The negative association between gamma and age has previously been observed in this age range [42]. Larger studies in this young age range are needed to better understand both the developmental course of EEG measures in this population, and their relation to core symptoms. Second, this analysis did not include EEG collected as part of a language or auditory based task. Without a stimulus input, it is unclear what narrowband resting-state beta/gamma activity represents. Increase EEG beta activity has been observed in response to $\mathrm{GABA}_{\mathrm{A}}$ receptor modulation, especially in the upper range $(21-30 \mathrm{~Hz})$, and this effect in rats is even further increased when animals are sitting or walking [68]. Increases in EEG measured beta and gamma power have also been associated with muscle artifact [69], however when this occurs measure increases are usually topographically specific to frontal or temporal regions. Here, the shift in beta/gamma peak and increased amplitude was similarly observed across all brain regions (Additional file 1: Figure 3). Interestingly, robust increases in spontaneous frontocentral beta oscillations (peaking at $23 \mathrm{~Hz}$ ) have also been observed in children with Dup15q syndrome [70]. Further investigation of what mechanistically drives $\sim 30 \mathrm{~Hz}$ oscillations may shed further light on the pathophysiology of FXS.

\section{Limitations and future directions}

The sample size of this study is small. Not surprisingly, recruitment of participants with rare genetic disorders is challenging and ultimately large studies will require collaboration and coordination across multiple sites. Further, collecting EEG data in young children with FXS can be difficult as they have significant behavioral challenges including sensitivity to be touched (especially on their heads), limited expressive language, difficulty sitting in one place, reduced attention, and challenges following directions. This study demonstrates that while EEG acquisition in this age group is feasible, 12/16 (75\%) participants cooperated with EEG net placement, success requires a research team experienced in both EEG acquisition and behavioral management of challenging behaviors.

Given the above behavioral challenges, consistency in behavior across participants during EEG acquisition was reduced. While most age-matched typically developing children watched the screen saver during baseline EEG acquisition, FXS participants' attention to the screen was reduced and sometimes required redirection or use of other non-social distractors. It is possible that differences between groups and between individuals are related to this variability in behaviors and emotional states. We do note that our cognitive-matched group was composed of largely toddler-aged boys (14-37 months old), who we expect to have age-appropriate challenges with attention and behavior. In addition, EEG quality metrics were similar across groups. Finally, pre-clinical mouse studies have observed increased gamma power in Fmr1 KO mice during periods of no movement [19], suggesting that these findings now observed in children, adults, and mice with FXS are reliable and robust.

\section{Conclusions}

Baseline EEG measures from this study in preschool age boys similarly identified gamma abnormalities previous documented in adults and mouse models. However, the unexpected relationship of these EEG abnormalities to clinical symptoms, highlights the complexity of neurodevelopmental disorders such as FXS and the need for larger studies across early childhood in order to further understand changes in EEG measures across development and how these measures relate to clinical symptoms and outcomes.

\section{Supplementary Information}

The online version contains supplementary material available at https://doi. org/10.1186/s13229-021-00425-x.

Additional file 1. Supplemental Figures and Tables.

\section{Abbreviations}

ABC-FXS: Aberrant Behavior Checklist-Community Edition; FXS scoring; ASD: Autism spectrum disorder; BEAPP: Batch EEG Automated Processing Platform; EEG: Electroencephalography; EGI: Electrical Geodesics, Inc; FMRP: Fragile X Mental Retardation Protein; FOOOF: Fitting Oscillations and One-Over-F; FXS: Fragile X Syndrome; HAPPE: Harvard Automated Preprocessing Pipeline for EEG; MARA: Multiple Artifact Rejection Algorithm; MSEL: Mullen Scales of Early Learning; NVDQ: Nonverbal Developmental Quotient; PSD: Power spectral density; PLS-5: Preschool Language Scale, 5th Edition; VABS-3: Vineland Adaptive Behavior Scales, 3rd Edition.

\section{Acknowledgements}

We thank all the families who participated in this study. We also thank Jack Keller, Megan Lauzé, John Fitzgerald, Megan Hartney and the Translational Neuroscience Center Human Neurobehavioral Core at Boston Children's for their assistance in data collection of FXS and age-matched controls. We thank the Infant Screening Study Team for their assistance in data collection of the cognitive-matched controls. We also thank Graham Holt for his EEG technical support.

\section{Authors' contributions}

CLW contributed to study conception and design, EEG data acquisition, EEG and behavioral data analysis, data interpretation and statistical analysis, and drafting of the manuscript. CAN contributed to the study design, overseeing data acquisition, and critically reviewed the manuscript for intellectual content. Both authors read and approved the final manuscript.

\section{Funding}

Support for this work was provided by: FRAXA Research Foundation, Autism Science Foundation, The Pierce Family Fragile X Foundation, Thrasher Research Fund, Society for Developmental Behavioral Pediatrics, Harvard Catalyst 
Medical Research Investigator Training Award, and the National Institutes of Health (1T32MH112510, 1K23DC017983-01A1, and R01DC010290).

\section{Availability of data and materials}

The data sets used and/or analyzed during the current study are available from the corresponding author upon reasonable request.

\section{Ethics approval and consent to participate}

Institutional review board approval was obtained from Boston Children's Hospital (IRB\#P00025493, IRB\#P00018377) prior to starting the study. Written, informed consent was obtained from all caregivers prior to their children's participation in the study.

\section{Consent for publication}

Not applicable.

\section{Competing interests}

The authors declare that they have no conflicts of interest.

Received: 14 October 2020 Accepted: 16 February 2021

Published online: 25 February 2021

\section{References}

1. Hersh JH, Saul RA, Saal HM, Braddock SR, Enns GM, Gruen JR, et al. Clinical report-health supervision for children with fragile $X$ syndrome. Pediatrics. 2011;127:994-1006. https://doi.org/10.1542/peds.2010-3500.

2. Hagerman RJ, Berry-Kravis E, Kaufmann WE, Ono MY, Tartaglia N, Lachiewicz $A$, et al. Advances in the treatment of fragile $X$ syndrome. Pediatrics. 2009;123(1):378-90.

3. Hernandez RN, Feinberg RL, Vaurio R, Passanante NM, Thompson RE, Kaufmann WE. Autism spectrum disorder in fragile $X$ syndrome: a longitudinal evaluation. Am J Med Genet Part A. 2009;149A(6):1125-37.

4. Rogers SJ, Wehner EA, Hagerman R. The behavioral phenotype in fragile $X$ : Symptoms of autism in very young children with fragile $X$ syndrome, idiopathic autism, and other developmental disorders. J Dev Behav Pediatr. 2001;22(6):409-17.

5. Sidorov MS, Auerbach BD, Bear MF. Fragile $X$ mental retardation protein and synaptic plasticity. Mol Brain. 2013;6(1):15.

6. Gibson JR, Bartley AF, Hays SA, Huber KM. Imbalance of neocortical excitation and inhibition and altered UP states reflect network hyperexcitability in the mouse model of fragile X syndrome. J Neurophysiol. 2008;100(5):2615-26.

7. Lozano R, Hare E, Hagerman R. Modulation of the GABAergic pathway for the treatment of fragile X syndrome. Neuropsychiatr Dis Treat. 2014;10:1769.

8. Contractor A, Klyachko VA, Portera-Cailliau C. Altered neuronal and circuit excitability in fragile X syndrome. Neuron. 2015;87(4):699-715. https:// doi.org/10.1016/j.neuron.2015.06.017.

9. Antoine MW, Langberg T, Schnepel P, Feldman DE. Increased excitationinhibition ratio stabilizes synapse and circuit excitability in four autism mouse models. Neuron. 2019;101(4):648-661.e4.

10. Michalon A, Sidorov M, Ballard TM, Ozmen L, Spooren W, Wettstein JG, et al. Chronic pharmacological mGlu5 inhibition corrects fragile $X$ in adult mice. Neuron. 2012;74(1):49-56.

11. Henderson C, Wijetunge L, Kinoshita MN, Shumway M, Hammond RS, Postma FR, et al. Reversal of disease-related pathologies in the fragile $X$ mouse model by selective activation of GABAB receptors with arbaclofen. Sci Transl Med. 2012;4(152):152ra128. https://doi.org/10.1126/scitranslm ed.3004218.

12. Qin M, Huang T, Kader M, Krych L, Xia Z, Burlin T, et al. R-baclofen reverses a social behavior deficit and elevated protein synthesis in a mouse model of fragile X syndrome. Int J Neuropsychopharmacol. 2015;18(9):1-13.

13. Berry-Kravis E, Hessl D, Abbeduto L, Reiss AL, Beckel-Mitchener A, Urv TK, et al. Outcome measures for clinical trials in fragile $X$ syndrome. J Dev Behav Pediatr. 2013;34(7):508-22.

14. Levin AR, Nelson CA. Inhibition-based biomarkers for autism spectrum disorder. Neurotherapeutics. 2015;12:546-52. https://doi.org/10.1007/ s13311-015-0350-1.
15. Jacquemont S, Berry-Kravis E, Hagerman R, Von Raison F, Gasparini F, Apostol $G$, et al. The challenges of clinical trials in fragile $X$ syndrome. Psychopharmacology. 2014;231:1237-50.

16. Ethridge LE, De Stefano LA, Schmitt LM, Woodruff NE, Brown KL, Tran M, et al. Auditory EEG biomarkers in fragile X syndrome: clinical relevance. Front Integr Neurosci. 2019;13:60.

17. Wang J, Ethridge LE, Mosconi MW, White SP, Binder DK, Pedapati EV, et al. A resting EEG study of neocortical hyperexcitability and altered functional connectivity in fragile X syndrome. J Neurodev Disord. 2017;9(1):11.

18. Van Der Molen MJW, Van Der Molen MW. Reduced alpha and exaggerated theta power during the resting-state EEG in fragile $X$ syndrome. Biol Psychol. 2013;92:216-9.

19. Lovelace JW, Ethell IM, Binder DK, Razak KA. Translation-relevant EEG phenotypes in a mouse model of fragile $X$ syndrome. Neurobiol Dis. 2018;115:39-48. https://doi.org/10.1016/j.nbd.2018.03.012.

20. Wen TH, Lovelace JW, Ethell IM, Binder DK, Razak KA. Developmental changes in EEG phenotypes in a mouse model of fragile $X$ syndrome. Neuroscience. 2019;398:126-43. https://doi.org/10.1016/j.neuroscien ce.2018.11.047

21. Knoth IS, LajnefT, Rigoulot S, Lacourse K, Vannasing P, Michaud JL, et al. Auditory repetition suppression alterations in relation to cognitive functioning in fragile $X$ syndrome: a combined EEG and machine learning approach. J Neurodev Disord. 2018;10(1):4. https://doi.org/10.1186/s1168 9-018-9223-3.

22. Castrén M, Pääkkönen A, Tarkka IM, Ryynänen M, Partanen J. Augmentation of auditory $N 1$ in children with fragile $X$ syndrome. Brain Topogr. 2003:15(3):165-71.

23. Knoth IS, Lippé S. Event-related potential alterations in fragile $X$ syndrome. Front Hum Neurosci. 2012;6:264. https://doi.org/10.3389/fnhum .2012.00264.

24. Schneider A, Leigh MJ, Adams P, Nanakul R, Chechi T, Olichney J, et al. Electrocortical changes associated with minocycline treatment in fragile X syndrome. J Psychopharmacol. 2013;27(10):956-63. https://doi. org/10.1177/0269881113494105.

25. Sinclair D, Featherstone R, Naschek M, Nam J, Du A, Wright S, et al. GABA-B agonist baclofen normalizes auditory-evoked neural oscillations and behavioral deficits in the Fmr 1 knockout mouse model of fragile $X$ syndrome. eNeuro 2017;4(1)

26. Sohal VS, Rubenstein JLR. Excitation-inhibition balance as a framework fo investigating mechanisms in neuropsychiatric disorders. Mol Psychiatry. 2019;24(9):1248-57.

27. Wen TH, Afroz S, Reinhard SM, Palacios AR, Tapia K, Binder DK, et al. Genetic reduction of matrix metalloproteinase-9 promotes formation of perineuronal nets around parvalbumin-expressing interneurons and normalizes auditory cortex responses in developing Fmr1 knock-out mice. Cereb Cortex. 2018;28(11):3951-64.

28. Hirano Y, Oribe N, Kanba S, Onitsuka T, Nestor PG, Spencer KM. Spontaneous gamma activity in schizophrenia. JAMA Psychiatry. 2015;72(8):813-21.

29. Roach BJ, Mathalon DH. Event-related EEG time-frequency analysis: an overview of measures and an analysis of early gamma band phase locking in schizophrenia. Schizophr Bull. 2008;34(5):907-26.

30. Gonzalez-Burgos G, Cho RY, Lewis DA. Alterations in cortical network oscillations and parvalbumin neurons in schizophrenia. Biol Psychiatry. 2015;77(12):1031-40.

31. Rojas DC, Wilson LB. $y$-band abnormalities as markers of autism spectrum disorders. Biomark Med. 2014;8(3):353-68.

32. Gabard-Durnam LJ, Wilkinson C, Kapur K, Tager-Flusberg H, Levin AR, Nelson CA. Longitudinal EEG power in the first postnatal year differentiates autism outcomes. Nat Commun. 2019;10(1):4188. https://doi. org/10.1038/s41467-019-12202-9.

33. Gogolla N, Leblanc JJ, Quast KB, Südhof TC, Fagiolini M, Hensch TK. Common circuit defect of excitatory-inhibitory balance in mouse models of autism. J Neurodev Disord. 2009;1(2):172-81.

34. Mcfadden KL, Hepburn S, Winterrowd E, Schmidt GL, Rojas DC. Abnormalities in gamma-band responses to language stimuli in first-degree relatives of children with autism spectrum disorder: an MEG study. BMC Psychiatry. 2012;12:1.

35. Peña $M$, Pittaluga $E$, Mehler J. Language acquisition in premature and full-term infants. Proc Natl Acad Sci USA. 2010;107:3823-8.

36. Wilkinson $C L$, Levin AR, Gabard-Durnam $L$, Tager-Flusberg $H$, Nelson CA. Reduced frontal gamma power at 24 months is associated with 
better expressive language in toddlers at risk for autism. Autism Res. 2019;12:1211-24.

37. Benasich AA, Gou Z, Choudhury N, Harris KD. Early cognitive and language skills are linked to resting frontal gamma power across the first 3 years. Behav Brain Res. 2008;195:215-22.

38. Gou Z, Choudhury N, Benasich AA. Resting frontal gamma power at 16, 24 and 36 months predicts individual differences in language and cognition at 4 and 5 years. Behav Brain Res. 2011;220(2):263-70.

39. Howard MW, Rizzuto DS, Caplan JB, Madsen JR, Lisman J, AschenbrennerScheibe R, et al. Gamma oscillations correlate with working memory load in humans. Cereb Cortex. 2003;13(12):1369-74. https://doi.org/10.1093/ cercor/bhg084.

40. Pesaran B, Pezaris JS, Sahani M, Mitra PP, Andersen RA. Temporal structure in neuronal activity during working memory in macaque parietal cortex. Nat Neurosci. 2002;5(8):805-11.

41. Schmitt LM, Wang J, Pedapati EV, Thurman AJ, Abbeduto L, Erickson CA, et al. A neurophysiological model of speech production deficits in fragile X syndrome. Brain Commun. 2020;2(1):fcz042. https://doi.org/10.1093/ braincomms/fcz042/5670524.

42. Orekhova EV, Stroganova TA, Nygren G, Tsetlin MM, Posikera IN, Gillberg C, et al. Excess of high frequency electroencephalogram oscillations in boys with autism. Biol Psychiatry. 2007:62(9):1022-9.

43. Ferree TC, Luu P, Russell GS, Tucker DM. Scalp electrode impedance, infection risk, and EEG data quality. Clin Neurophysiol. 2001;112(3):536-44.

44. Levin AR, Méndez Leal AS, Gabard-Durnam LJ, O'Leary HM. BEAPP: the batch electroencephalography automated processing platform. Front Neurosci. 2018;12:513.

45. Gabard-Durnam LJ, Mendez Leal AS, Wilkinson CL, Levin AR. The Harvard Automated Processing Pipeline for Electroencephalography (HAPPE): standardized processing software for developmental and high-artifact data. Front Neurosci. 2018:12:97.

46. Winkler I, Haufe S, Tangermann M. Automatic classification of artifactual ICA-components for artifact removal in EEG signals. Behav Brain Funct. 2011;7(1):30.

47. Winkler I, Brandl S, Horn F, Waldburger E, Allefeld C, Tangermann M. Robust artifactual independent component classification for $\mathrm{BCl}$ practitioners. J Neural Eng. 2014;11(3):035013.

48. Thomson DJ. Spectrum estimation and harmonic analysis. Proc IEEE. 1982;70(9):1055-96.

49. Donoghue T, Haller M, Peterson EJ, Varma P, Sebastian P, Gao R, et al. Parameterizing neural power spectra into periodic and aperiodic components. Nat Neurosci. 2020;23(12):1655-65.

50. Mullen E. Infant Mullen Scales of Early Learning (Infant MSEL). TOTAL Child, Incorporated; 1989. http://www.pearsonclinical.com/childhood/ products/100000306/mullen-scales-of-early-learning.html\#tab-details

51. Zimmerman IL, Steiner VG, Pond RE. Preschool Language Scales, Fifth Edition. Fifth. Pearson Education, Inc; 2011. http://www.pearsonclinical.com/ language/products/100000233/preschool-language-scales-fifth-editi on-pls-5.html

52. Aman MG, Singh NN, Stewart AW, Field CJ. The aberrant behavior checklist: a behavior rating scale for the assessment of treatment effects. Am J Ment Defic. 1985;89(5):485-91.

53. Sansone SM, Widaman KF, Hall SS, Reiss AL, Lightbody A, Kaufmann WE, et al. Psychometric study of the aberrant behavior checklist in fragile $X$ syndrome and implications for targeted treatment. J Autism Dev Disord. 2012;42(7):1377-92
54. Sparrow S. Vineland Adaptive Behavior Scales. Third. The SAGE Encyclopedia of Intellectual and Developmental Disorders. London: Pearson Education, Inc; 2018.

55. Lam KSL, Aman MG. The Repetitive Behavior Scale-Revised: independent validation in individuals with autism spectrum disorders. J Autism Dev Disord. 2007;37(5):855-66.

56. Dunn W. Sensory Profile 2. London: Pearson Education, Inc; 2014.

57. Maris E, Oostenveld R. Nonparametric statistical testing of EEG-and MEGdata. J Neurosci Methods. 2007:164:177-90.

58. Gramfort A, Luessi M, Larson E, Engemann DA, Strohmeier D, Brodbeck $C$, et al. MNE software for processing MEG and EEG data. Neuroimage. 2014:86:446-60.

59. Hunter JD. Matplotlib: a 2D graphics environment. Comput Sci Eng 2007:9(3):99-104.

60. Gao R, Peterson EJ, Voytek B. Inferring synaptic excitation/inhibition balance from field potentials. Neuroimage. 2017. https://doi.org/10.1016/j. neuroimage.2017.06.078.

61. Voytek B, Knight RT. Dynamic network communication as a unifying neural basis for cognition, development, aging, and disease. Biol Psychiatry. 2015:77(12):1089-97.

62. He BJ, Zempel JM, Snyder AZ, Raichle ME. The temporal structures and functional significance of scale-free brain activity. Neuron. 2010;66(3):353-69.

63. Olmos-Serrano JL, Paluszkiewicz SM, Martin BS, Kaufmann WE, Corbin JG, Huntsman MM. Defective GABAergic neurotransmission and pharmacological rescue of neuronal hyperexcitability in the amygdala in a mouse model of fragile X syndrome. J Neurosci. 2010;30(29):9929-38.

64. Selby L, Zhang C, Sun QQ. Major defects in neocortical GABAergic inhibitory circuits in mice lacking the fragile $\mathrm{X}$ mental retardation protein. Neurosci Lett. 2007:412(3):227-32.

65. Goel A, Portera-Cailliau C. Autism in the balance: elevated E-I ratio as a homeostatic stabilization of synaptic drive. Neuron. 2019;101:543-5. https://doi.org/10.1016/j.neuron.2019.01.033.

66. Ethridge LE, White SP, Mosconi MW, Wang J, Pedapati EV, Erickson CA, et al. Neural synchronization deficits linked to cortical hyper-excitability and auditory hypersensitivity in fragile X syndrome. Mol Autism. 2017;8(1):22.

67. De Stefano LA, Schmitt LM, White SP, Mosconi MW, Sweeney JA, Ethridge LE. Developmental effects on auditory neural oscillatory synchronization abnormalities in autism spectrum disorder. Front Integr Neurosci. 2019;13:34.

68. Van Lier H, Drinkenburg WHIM, Van Eeten YJW, Coenen AML. Effects of diazepam and zolpidem on EEG beta frequencies are behavior-specific in rats. Neuropharmacology. 2004;47(2):163-74.

69. Goncharova I, McFarland D, Vaughan T, Wolpaw J. EMG contamination of EEG: spectral and topographical characteristics. Clin Neurophysiol. 2003:114(9):1580-93.

70. Frohlich J, Reiter LT, Saravanapandian V, DiStefano C, Huberty S, Hyde C, et al. Mechanisms underlying the EEG biomarker in Dup15a syndrome. Mol Autism. 2019;10(1):29. https://doi.org/10.1186/s13229-019-0280-6.

\section{Publisher's Note}

Springer Nature remains neutral with regard to jurisdictional claims in published maps and institutional affiliations.

Ready to submit your research? Choose BMC and benefit from:

- fast, convenient online submission

- thorough peer review by experienced researchers in your field

- rapid publication on acceptance

- support for research data, including large and complex data types

- gold Open Access which fosters wider collaboration and increased citations

- maximum visibility for your research: over 100M website views per year

At BMC, research is always in progress.

Learn more biomedcentral.com/submissions 